CLAWAR 2020: 23rd International Conference on

Climbing and Walking Robots and the Support

Technologies for Mobile Machines,

Moscow, Russian Federation, 24-26 August 2020.

https://doi.org/10.13180/clawar.2020.24-26.08.56

\title{
The DLS Quadruped Proprioceptive Sensor Dataset
}

\author{
Geoff Fink and Claudio Semini \\ Dynamic Legged Systems Lab, Istituto Italiano di Tecnologia (IIT), Via Morego 30, \\ Genova, 16163, Italy \\ E-mail: \{geoff.fink,claudio.semini\}@iit.it \\ https://dls.iit.it/
}

\begin{abstract}
This paper presents novel datasets of the hydraulically actuated robot HyQ's proprioceptive sensors. All of the datasets include absolute and relative joint encoders, joint force and torque sensors, and MEMS-based and fibre optic-based inertial measurement units (IMUs). Additionally, a motion capture system recorded the ground truth data with millimetre accuracy. In the datasets $\mathrm{HyQ}$ was manually controlled to trot in place or move around the laboratory. The sequence includes: forward and backwards motion, side-to-side motion, zig-zags, yaw motion, and a mix of linear and yaw motion. Additionally, there is motion on both rigid and soft terrain. All of the datasets are at least five minutes long and one dataset is thirty minutes long. The aim of these datasets is to test, evaluate, and compare different algorithms for state estimation using only proprioceptive sensors. To aid in the development of new state estimation algorithms for soft terrain there are datasets comparing rigid to soft terrain. Lastly, the extra long endurance trot dataset is for examining the problem of long-term attitude estimation drift.

The datasets can be downloaded from https://www.doi.org/10.21227/4vxz-xw05.
\end{abstract}

Keywords: Dataset, Proprioceptive Sensors, Legged Locomotion, Quadruped Robots

\section{Introduction}

Quadruped robots are gaining increasing attention in numerous research areas. ${ }^{1-4}$ They are advantageous when compared to wheeled or tracked robots in that they are more versatile and can navigate uneven and rough terrain easier. They are able to achieve this by having more degrees of freedom. However, these new abilities come coupled with increased mechanical complexity and increased difficulty in state estimation and control.

The state of a robot is a set of quantities that describes pertinent information about the robot. Typically, for a mobile robot the state will include the position and velocity. Furthermore, it can include information such as forces, torques, centre of masses, or even $3 \mathrm{D}$ maps. State estimation is the problem of estimating the state of a robot from sensor data and models. The difficulty of state estimation is often underestimated. Controlling a robot can be relatively easy if the pose of the robot and a map of the environment are known. However, there are no sensors that can directly measure these variables, and the sensors that can partially measure them are corrupted with noise. Further details regarding state estimation for mobile robotics can be found in Ref. 5 and Ref. 6.

The literature on state estimation for mobile robotics can be divided into different tasklevels. Much of the current literature has been focused on exteroceptive sensors for simultaneous localization and mapping (SLAM), ${ }^{7}$ visual odometry ${ }^{8}$ visual SLAM, ${ }^{9}$ and visualinertial SLAM. ${ }^{10-12}$ The goal of these works is to provide a non-drifting pose for the task of robot navigation, however, the main drawbacks of these approaches are the frequencies and delays in many of the sensors used, e.g., cameras and lidars. There is also a less numerous, but growing body of literature for state estimation of legged robots for the task of locomotion. ${ }^{13-16}$ Similar to the SLAM literature these works suffer from the slow update rates of the aiding sensors, such as lidars and cameras. Lastly, there is also literature for lower level state estimation using only proprioceptive sensors. ${ }^{2,17-20}$ Ref. 20 uses datasets 


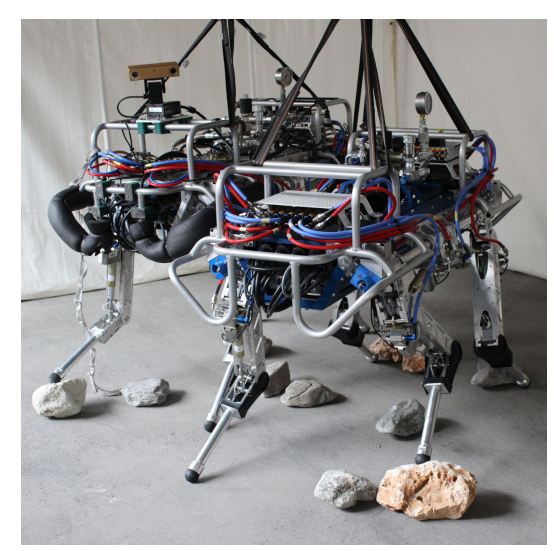

Fig. 1. A photo of two HyQ quadruped robots.

from this paper.

There are many difficulties in comparing results from attitude state estimators across different platforms. The obvious obstacle is the hardware: different sensor accuracies, different sensor frequencies, different dynamics, different actuation, different locomotion strategies, and different operating environments can have large impacts on the quality of the state estimate. Apart from the hardware, the software also affects the estimate. Different implementations of the same algorithm can cause large numeric errors in floating point math; the processor speed and operating system choice can cause large time-varying delays in the sensors readings. Lastly, even on two identical platforms different sensor calibrations may also have a large impact on state estimation. Errors in the transformation from the sensor frame $\mathcal{S}$ to the body frame $\mathcal{B}$ can cause divergence of some sensor fusion algorithms. To help overcome these difficulties, one of the goals of this work is an attempt to standardize and share datasets for quadruped robots similar to what is currently being done in the visual-inertial SLAM community. ${ }^{21-24}$

There are two main solutions to overcome these problems of comparing datasets: standardized benchmarks and standardized datasets. Multiple benchmarks to compare odometry and state estimator algorithms have been proposed for visual-inertial odometry and SLAM. ${ }^{21,22,25}$ Many of these tools can be directly applied to quadruped odometry. Similarly, many datasets have been published for visual and visual-inertial odometry. ${ }^{23,24,26}$ However, these datasets do not contain the typical sensors that are present in quadrupeds, i.e., force and torque sensors; absolute and relative joint encoders. To the best of the authors' knowledge similar datasets for quadruped robots that contain proprioceptive sensors do not exist in the public domain.

This paper is an extension of a field report presented at a recent workshop conference. ${ }^{27}$ The main contribution of this paper is a novel dataset collected on board the hydraulically actuated quadruped HyQ. ${ }^{1}$ The dataset contains all of HyQ's proprioceptive sensors running at high frequencies in multiple scenarios. Furthermore, it was collected in a laboratory that has millimetre accurate position ground truth.

\section{Experimental Setup}

The experimental platform for these dataset is the robot HyQ. HyQ weighs approximately $90 \mathrm{~kg}$ depending on its current sensor suite and has twelve torque-controlled joints powered by hydraulic actuators. The hydraulic actuators allow the robot to perform powerful dynamic motions. Detailed mechanical specifications for HyQ are in Ref. 1 and Ref. 28. In this section we highlight some of the relevant geometric parameters of the legs and the joints that are 


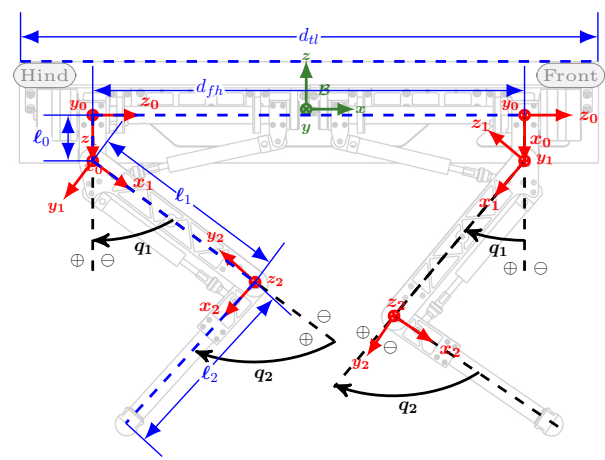

(a) Right Side View

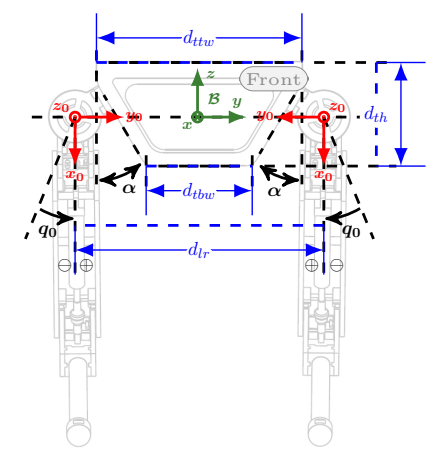

(b) Front View

Fig. 2. Diagram of HyQ showing the geometric parameters, the reference frame of the body $\mathcal{B}=\{x, y, z\}$, and the basis of the joints $\left\{x_{i}, y_{i}, z_{i}\right\}$ where $i=\{0,1,2\}=\{$ HAA, HFE, KFE $\}$. The left side is a mirror copy of the right side. The parameters are defined in Table 1.

necessary for calculating kinematics of the robot.

We consider a floating base robot with four feet and three DoFs per leg. The four legs are labelled left front (LF), right front $(\mathrm{RF})$, left hind $(\mathrm{LH})$, and right hind $(\mathrm{RH})$. Each leg has three actuated joints: hip abduction/adduction (HAA), Hip flexion/extension (HFE), and knee flexion/extension (KFE).

$$
\begin{aligned}
& \ell \in \mathbb{L}=\{\mathrm{LF}, \mathrm{RF}, \mathrm{LH}, \mathrm{RH}\} \\
& j \in \mathbb{J}=\{\mathrm{HAA}, \mathrm{HFE}, \mathrm{KFE}\} \\
& i \in \mathbb{I}=\mathbb{L} \times \mathbb{J}
\end{aligned}
$$

Also labelled in the figure are the lengths between each joint. The body frame $\mathcal{B}$ of the robot is located at the geometric centre of the four HAA joints and is orientated such that the $x$, $y, z$ axes are pointing forward, left, and up, respectively. The geometric parameters of HyQ are in Table 1.

There are three important types of proprioceptive sensors on board HyQ: joint encoders, force/torque sensors, and IMUs. Each joint contains an absolute and relative encoder to measure the joint angle. The absolute encoder (AMS Programmable Magnetic Rotary Encoder - AS5045) is used to measure the joint position when the robot is first turned on. Next, the relative encoder (Avago Ultra Miniature, High Resolution Incremental Encoder - AED-3300-TE1) is used to measure how far the joint has moved at every epoch. Also, every joint contains a force or torque sensor. In both the KFE and the HFE there is a load cell (Burster Subminiature Load Cell - 8417-6005 ) and the HAA has a custom designed torque sensor based on strain-gauges and is similar to Ref. 29. In the trunk of the robot there are two IMUs: a military grade fibre optic KVH-1775 and a MEMS-based high-end consumer grade Lord MicroStrain 3DM-GX5-15. One of the goals of this dataset is to provide an opportunity for researchers to compare the two IMUs using state-of-the-art state estimation algorithms to determine how much the increased accuracy affects drift and if the accuracy is worth the extra cost, size, and weight. All of the sensors are rigidly attached to HyQ with the exception of the 3DM-GX5-15. It has been mounted on top of a $6 \mathrm{~mm}$ thick Sorbothane vibration isolation pad as suggested by the manufacture. We note that this is not necessary for the fibre optic IMU. The basis vectors of the GX4 sensor frame are orientated forward, right, and down; and the basis vectors of the KVH sensor frame are orientated right, forward, and down. To measure the ground truth pose of the robot the lab is equipped with a motion capture system (MCS). In particular we use a mix of Vicon T10 and Vero 2 cameras. A summary of the proprioceptive sensors on board HyQ are in Table 2. 
Table 1. Geometric parameters of the legs and joint kinematics.

\begin{tabular}{|lccc|}
\hline Location & Parameter & Value & Units \\
\hline trunk & $\left(d_{\mathrm{t} t} d_{\mathrm{th}} d_{\mathrm{ttw}}\right)$ & $(1000180340)$ & $\mathrm{mm}$ \\
& $\left(d_{\mathrm{fh}} d_{\mathrm{lr}}\right)$ & $(747414)$ & $\mathrm{mm}$ \\
& $d_{\mathrm{tbw}}$ & 85 & $\mathrm{~mm}$ \\
leg & $\alpha$ & 30 & $\mathrm{~mm}$ \\
& $l_{0}$ & 80 & $\mathrm{~mm}$ \\
& $l_{1}$ & 350 & $\mathrm{~mm}$ \\
HAA & $l_{2}$ & 346 & $\mathrm{~mm}$ \\
HFE (left) & $l_{3}$ & 20 & $\circ$ \\
HFE (right) & $q_{0}$ & $-70 \leq q_{0} \leq 25$ & $\circ$ \\
KFE (front) & $q_{1}$ & $-50 \leq q_{1} \leq 70$ & $\circ$ \\
KFE (back) & $q_{1}$ & $-70 \leq q_{1} \leq 50$ & $\circ$ \\
\hline
\end{tabular}

Table 2. Technical specifications of the proprioceptive sensors.

\begin{tabular}{|c|c|c|c|}
\hline \multicolumn{4}{|c|}{ Encoders } \\
\hline & AMS Rotary Encoder & Avago Encoder & \\
\hline Resolution & 4096 & 80000 & cpr \\
\hline Accuracy & \pm 0.5 & \pm 0.5 & $\operatorname{deg}$ \\
\hline Max. Speed & 153 & 1950 & rpm \\
\hline Max. Sampling Freq. & 10 & 650 & $\mathrm{kHz}$ \\
\hline \multicolumn{4}{|c|}{ Force/Torque Sensors } \\
\hline & Burster Load Cell & IIT-DLS Torque Sensor & \\
\hline Range & \pm 5000 & \pm 200 & $\mathrm{~N}, \mathrm{Nm}$ \\
\hline \multicolumn{4}{|c|}{ Inertial Measurement Units (IMUs) } \\
\hline & KVH 1775 IMU & Microstrain GX5 & \\
\hline Technology & Fibre optics & MEMS & - \\
\hline \multicolumn{4}{|c|}{ Gyroscope } \\
\hline Input Limit & 490 & 300 & $\mathrm{deg} / \mathrm{sec}$ \\
\hline Typical Bias Instability & 0.05 & 8 & $\operatorname{deg} / \mathrm{hr}$ \\
\hline Random Walk & 0.7 & 18 & $\frac{\mathrm{deg}}{\mathrm{hr} \sqrt{\mathrm{Hz}}}$ \\
\hline Bandwidth & 440 & 250 & $\mathrm{~Hz}$ \\
\hline Max. Sampling Freq. & 5000 & 4000 & $\mathrm{~Hz}$ \\
\hline \multicolumn{4}{|c|}{ Accelerometer } \\
\hline Input Limit & \pm 10 & \pm 8 & g \\
\hline Typical Bias Instability & 0.05 & 0.04 & $\mathrm{mg}$ \\
\hline Random Walk & 120 & 25 & $\frac{\mu \mathrm{g}}{\sqrt{\mathrm{Hz}}}$ \\
\hline Bandwidth & 200 & 225 & $\mathrm{~Hz}$ \\
\hline Max. Sampling Freq. & 5000 & 1000 & $\mathrm{~Hz}$ \\
\hline
\end{tabular}

Table 3. Comparison of datasets

\begin{tabular}{|c|c|c|c|c|c|c|}
\hline \multirow[b]{2}{*}{ Name } & \multirow{2}{*}{\begin{tabular}{|c|} 
Dur. \\
$t$ \\
$(\mathrm{~s})$ \\
\end{tabular}} & \multicolumn{2}{|c|}{ Distance } & \multicolumn{3}{|c|}{ Speed } \\
\hline & & $\begin{array}{c}\operatorname{sum}(|\Delta p|) \\
(\mathrm{m})\end{array}$ & $\begin{array}{c}\operatorname{sum}(\Delta p) \\
(\mathrm{m})\end{array}$ & $\begin{array}{c}\operatorname{mean}(|\Delta p| / d t) \\
(\mathrm{m} / \mathrm{s})\end{array}$ & $\begin{array}{c}\max (|\Delta p| / d t) \\
(\mathrm{m} / \mathrm{s})\end{array}$ & $\begin{array}{c}\operatorname{std}(|\Delta p| / d t) \\
(\mathrm{cm} / \mathrm{s})\end{array}$ \\
\hline Trot in Place & 349 & $\left(\begin{array}{lll}24 & 8 & 7\end{array}\right)$ & $\left(\begin{array}{lll}0.0 & 0.1 & 0.0\end{array}\right)$ & $\left(\begin{array}{lll}0.1 & 0.0 & 0.0\end{array}\right)$ & $\left(\begin{array}{lll}0.4 & 0.2 & 0.1\end{array}\right)$ & $\left(\begin{array}{lll}5 & 2 & 2\end{array}\right)$ \\
\hline Trot in Lab 1 & 374 & $\left(\begin{array}{lll}36 & 72 & 9\end{array}\right)$ & $\left(\begin{array}{lll}0.1 & 0.3 & 0.0\end{array}\right)$ & $\left(\begin{array}{lll}0.1 & 0.2 & 0.0\end{array}\right)$ & $\left(\begin{array}{lll}0.7 & 0.7 & 0.4\end{array}\right)$ & $\left(\begin{array}{lll}10 & 16 & 3\end{array}\right)$ \\
\hline Trot in Lab 2 & 344 & $\left(\begin{array}{lll}36 & 64 & 10\end{array}\right)$ & $\left(\begin{array}{lll}0.1 & 0.5 & 0.0\end{array}\right)$ & $\left(\begin{array}{lll}0.1 & 0.2 & 0.0\end{array}\right)$ & $\left(\begin{array}{lll}0.7 & 0.9 & 0.4\end{array}\right)$ & $\left(\begin{array}{lll}10 & 16 & 3\end{array}\right)$ \\
\hline Rigid Trot in Pla & 326 & $\left(\begin{array}{lll}14 & 18 & 9\end{array}\right)$ & $\left(\begin{array}{lll}0.0 & 0.2 & 0.0\end{array}\right)$ & $\left(\begin{array}{lll}0.0 & 0.1 & 0.0\end{array}\right)$ & $\left(\begin{array}{lll}0.5 & 0.3 & 0.2\end{array}\right)$ & $\left(\begin{array}{lll}3 & 4 & 2\end{array}\right)$ \\
\hline Soft Trot in Place & 322 & $\left(\begin{array}{lll}16 & 16 & 10\end{array}\right)$ & $\left(\begin{array}{lll}0.1 & 0.2 & 0.0\end{array}\right)$ & $\left(\begin{array}{lll}0,0 & 0.0 & 0,0\end{array}\right)$ & $\left(\begin{array}{lll}0.2 & 0.2 & 0.1\end{array}\right)$ & $\left(\begin{array}{lll}3 & 3 & 2\end{array}\right)$ \\
\hline Endurance Trot & 1817 & $\left(\begin{array}{lll}69 & 113 & 54\end{array}\right)$ & $\left(\begin{array}{lll}0.0 & 0.0 & 0.0\end{array}\right)$ & $\left(\begin{array}{lll}0.0 & 0.1 & 0.0\end{array}\right)$ & $\left(\begin{array}{lll}0.3 & 0.4 & 0.2\end{array}\right)$ & $\left(\begin{array}{lll}3 & 4 & 2\end{array}\right)$ \\
\hline
\end{tabular}


The first step in using any sensor is proper calibration. The force and torque sensors on board HyQ have external calibrations performed by the manufacturer before being installed. The absolute joint encoders must be calibrated after they are installed to a known angle. For this purpose we use a calibration frame that forces all of the legs into the position $q_{0}=q_{1}=0^{\circ}$ and $q_{2}=90^{\circ}$. The IMUs are also factory calibrated and do not have a specific offline calibration procedure, however, online bias states should be estimated, e.g., see Ref. 30. The last calibration procedure of importance is the (constant) transformations between the body frame, the IMU sensor frames, and the Vicon marker frame. For both of the IMUs the mounting position of the sensor is known in CAD except for an offset to the GX5 from the Sorbothane vibration isolation pad. The transformation to the Vicon marker frame is less accurate as it is a 3D printed plastic part with a higher tolerance.

The low level software framework runs on an Intel Intense PC 3 with Ubuntu 16 that has been compiled with a real-time kernel patch. The communication between all of the sensors and the actuators is based on EtherCAT. The master is programmed using SOEM. The slaves use an EtherCAT controller connected to a microcontroller. This architecture provides a high speed, low latency, and low jitter environment.

\section{Datasets}

The raw data from all of the datasets described in this paper can be found on the IEEE DataPort Platform ${ }^{31}$ with the following DOI: $10.21227 / 4 \mathrm{vxz}$-xw05. Each of the datasets was recorded on the quadruped robot HyQ indoors at the Dynamic Legged Systems laboratory located at the Istituto Italiano di Tecnologia (IIT) in Genoa, Italy. The aim of these datasets is to test state estimation using only proprioceptive sensors. For ground truth the lab is equipped with a MCS that records the pose of the robot with millimetre accuracy. Each of the datasets comprises of the raw sensor data of all the sensors listed in Table 2 recorded at $1000 \mathrm{~Hz}$ and the MCS ground truth recorded at $250 \mathrm{~Hz}$. The data is provided in both comma-separated values and Matlab file format. The data from the sensors and the data from MCS are on two distinct clocks. For convenience a plot of each of the sensors is provided online along side the numerical data.

HyQ has the ability to perform many dynamic gaits, for these datasets we chose to record trotting data as it is representative of a typical quadruped mission. In the Trot in Place dataset $\mathrm{HyQ}$ had to trot in place for more than five minutes. Occasional small corrections were given if HyQ drifted from its original position. In the Trot in Lab 1 \& 2 datasets HyQ was manually controlled by a joypad to trot around the laboratory. The sequence includes: forward and backwards motion, side-to-side motion, zig-zags, yaw motion, and a mix of linear, and yaw motion. Both datasets were also at least five minutes long. Soft terrain is a known problem for state estimators as it violate the common used assumption in leg odometry that the feet are static while in contact with the ground. ${ }^{32}$ To aid in the development of new state estimation algorithms for soft terrain there are the datasets Rigid Trot in Place and Soft Trot in Place. In the Soft Trot in Place HyQ is trotting on a foam block of $160 \times 120 \times 20 \mathrm{~cm}$. An indentation test of the foam shows the foam has an average stiffness of $2400 \mathrm{~N} / \mathrm{m}$. Lastly, to be able to see the slow drift in the IMU over a typical mission length, we recorded 30 minutes of trot data in the Endurance Trot dataset. The two jumps in the yaw at approximately $600 \mathrm{~s}$ an $1100 \mathrm{~s}$ are manual corrections sent via a remote control to the quadruped when it started to drift too far in one direction. It should be noted that there is a small gap in the ground truth data at approximately $1700 \mathrm{~s}$ for $39 \mathrm{~s}$.

We plot a subset of the dataset in Fig. 3-Fig. 7 due to space restrictions. Plots of all of the datasets are available online alongside the raw data. Fig. 3 and Fig. 4 show the joint positions and forces measured during the Trot in Place dataset. Fig. 5 shows the angular 


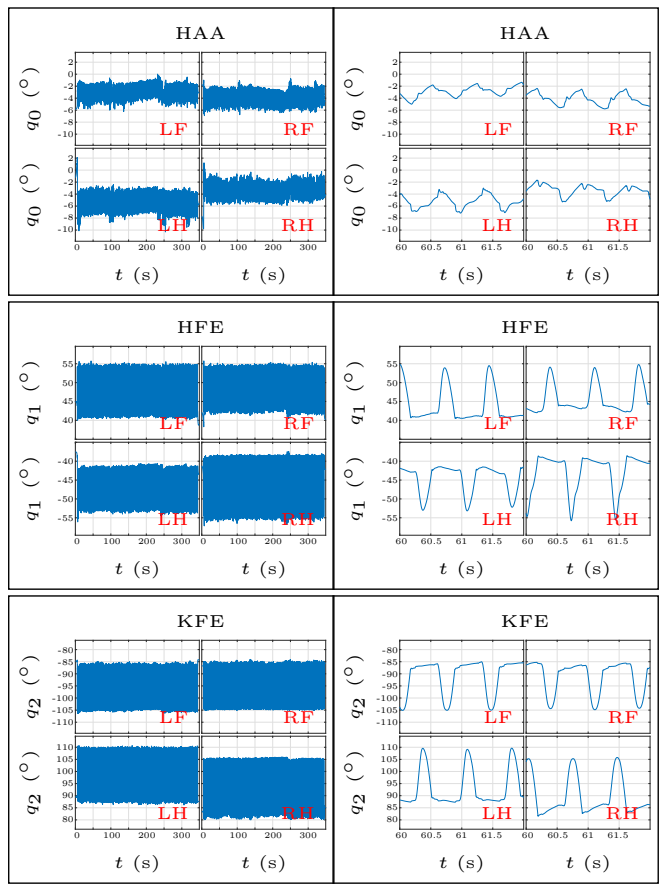

Fig. 3. The measured joint positions $q$ in the Trot in Place dataset.

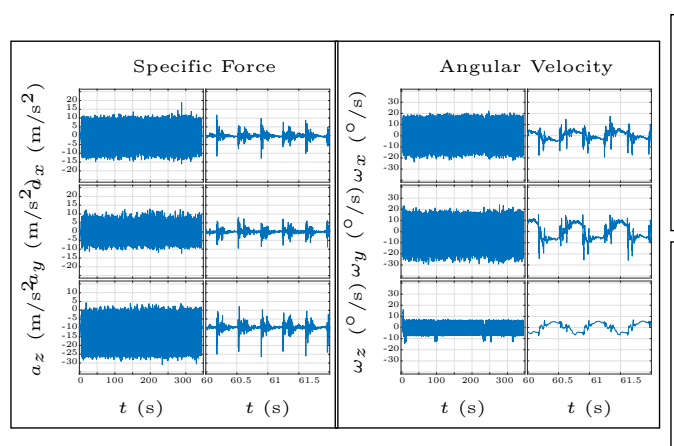

Fig. 5. The measured angular velocity $\omega$ and specific force $a$ in the sensor frames by the KVH for the Trot in Place dataset.

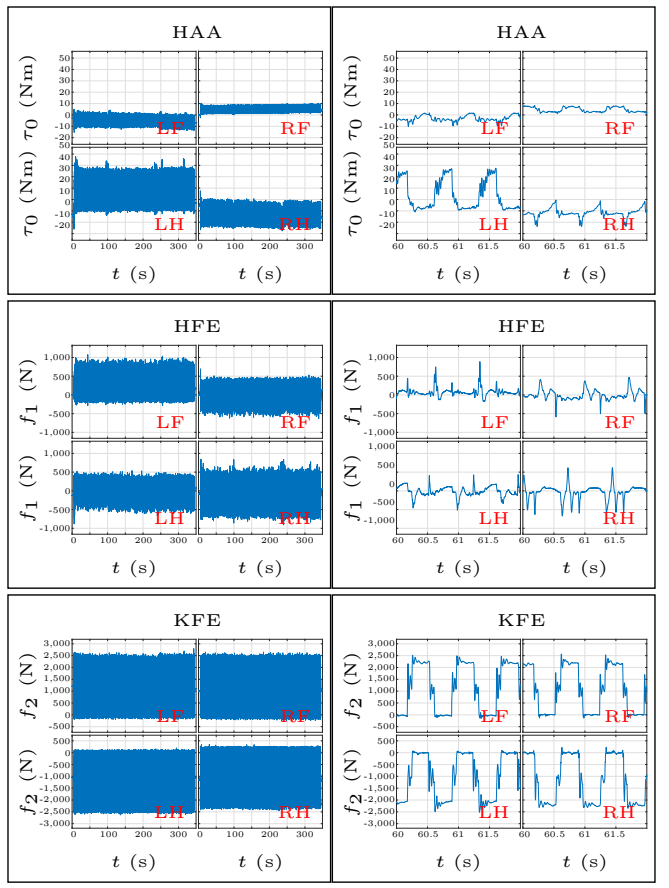

Fig. 4. The measured joint force $f$ and torque $\tau$ in the Trot in Place dataset.

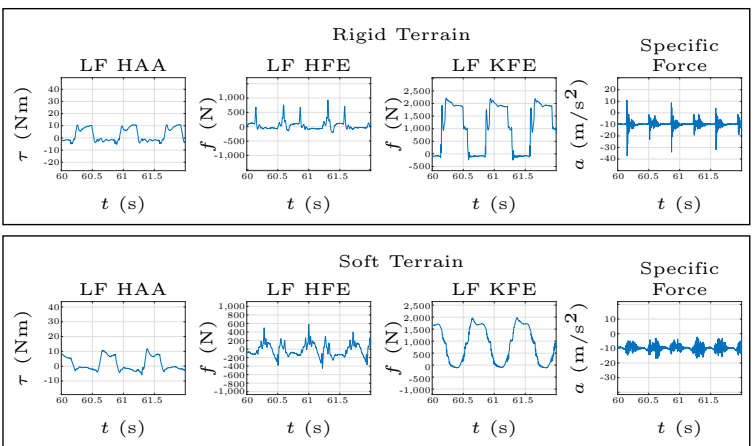

Fig. 6. The force and torque measurements for the LF leg during the Rigid Trot in Place versus Soft Trot in Place datasets.

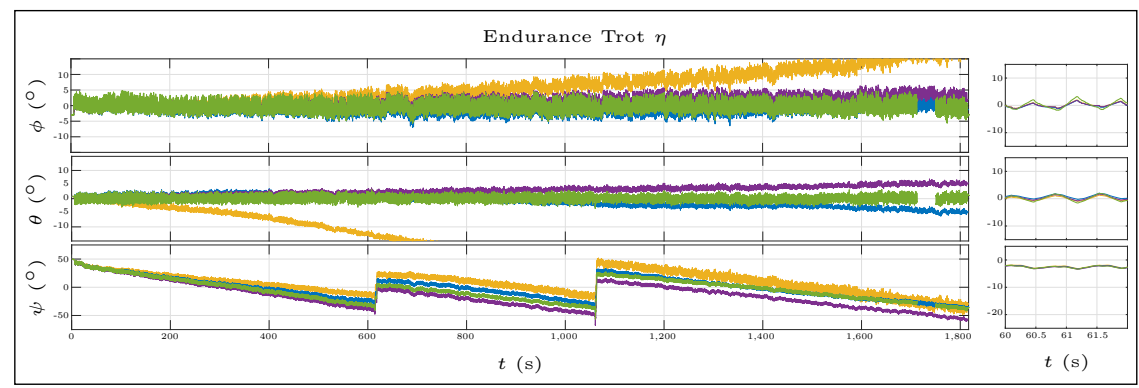

Fig. 7. The integral of the GX5 angular velocity with the bias removed and without the bias removed (blue and yellow, respectively), the integral of the $\mathrm{KVH}$ angular velocity (purple), and the ground truth from the MCS (green) for the Endurance Trot dataset. 
velocity and specific force measured by the KVH IMU during the same dataset. Fig. 3-Fig. 5 contain both an overview of the entire experiment and a zoomed in section at the one minute mark to more clearly view the signal. A summary of the datasets is shown in Table 3 .

\section{Discussion}

One of the important tasks of state estimation using proprioceptive sensors is attitude estimation. The simplest method to calculate attitude is to integrate the angular velocity starting at a known value. Note that we expect this value to drift due to the noise in the sensors and from bias instability. Furthermore, this value is a worst case scenario as it does not include sensor fusion nor known noise characteristics. However, it does provide a base comparison for the two IMUs used for the datasets. A comparison of the attitude provided by the ground truth (Vicon) and the estimated attitude via integration of the angular velocity by both IMUs is shown in Fig. 7. In the plot we denote the orientation using the XYZ Euler angles $\eta=[\phi, \theta, \psi]^{T}$. We expect this slow drift of both IMUs to decrease after sensor fusion. The plots also help demonstrate the difficulty in attitude estimation when there are large impacts and vibrations.

Another important topic in state estimation is the assumption of rigid terrain. In Fig. 6 we highlight some of the differences measured by the force sensors, torque sensors, and accelerometers when trotting on rigid terrain versus soft terrain. As can be seen through the KFE, the loading and unloading phases are a lot smoother on soft terrain. The robot's feet no longer have zero velocity while in contact with the ground. Further research in state estimation for quadrupeds is required to increase robustness and then to finally improve their autonomy.

\section{Conclusion}

In this work we presented novel datasets collected on board the hydraulically actuated quadruped HyQ. The datasets contain all of HyQ's proprioceptive sensors running at high frequencies for variety of scenarios. Additionally, the datasets contain precise ground truth data. We believe that these datasets will be highly useful to all researchers working on state estimation for quadruped robots and attitude estimation in general. Future work includes expanding the dataset to include more gaits and different terrains. Furthermore, we will use these datasets to benchmark our future state estimation algorithms.

\section{Acknowledgments}

We would like to thank all members of the DLS Lab for their valuable help. This research is funded in part by the Italian Workers' Compensation Authority (INAIL).

\section{References}

1. C. Semini, N. G. Tsagarakis, E. Guglielmino, M. Focchi, F. Cannella and D. G. Caldwell, Proceedings of the IMechE, Part I Journal of Systems and Control Engineering 225, 831 (2011).

2. G. Bledt, M. J. Powell, B. Katz, J. Di Carlo, P. M. Wensing and S. Kim, MIT Cheetah 3: Design and control of a robust, dynamic quadruped robot, in IEEE/RSJ International Conference on Intelligent Robots and Systems (IROS), (Madrid, Spain, 2018).

3. M. Hutter, C. Gehring, A. Lauber, F. Gunther, C. D. Bellicoso, V. Tsounis, P. Fankhauser, R. Diethelm, S. Bachmann, M. Bloesch, H. Kolvenbach, M. Bjelonic, L. Isler and K. Meyer, Advanced Robotics 31, 918 (2017).

4. M. Raibert, K. Blankespoor, G. Nelson and R. Playter, IFAC Proceedings Volumes 41, 10822 (2008).

5. S. Thrun, W. Burgard and D. Fox, Probabilistic Robotics (Intelligent Robotics and Autonomous Agents) (The MIT Press, Cambridge, MA, 2005). 
6. T. D. Barfoot, State Estimation for Robotics (Cambridge University Press, Cambridge, United Kingdom, 2017).

7. H. Durrant-Whyte and T. Bailey, IEEE Robotics and Automation Magazine 13, 99(June 2006).

8. D. Scaramuzza and F. Fraundorfer, IEEE Robotics and Automation Magazine 18, 80(December 2011).

9. A. J. Davison, I. D. Reid, N. D. Molton and O. Stasse, IEEE Transactions on Pattern Analysis and Machine Intelligence 29, 1052(June 2007).

10. A. Concha, G. Loianno, V. Kumar and J. Civera, Visual-inertial direct SLAM, in IEEE International Conference on Robotics and Automation (ICRA), (Stockholm, Sweden, 2016).

11. T. Pire, R. Baravalle, A. D'Alessandro and J. Civera, Robotica 36, 1510 (2018).

12. J. Engel, J. Sturm and D. Cremers, Robotics and Autonomous Systems 62, 1646 (2014), Special Issue on Visual Control of Mobile Robots.

13. S. Nobili, M. Camurri, V. Barasuol, M. Focchi, D. Caldwell, C. Semini and M. Fallon, Heterogeneous sensor fusion for accurate state estimation of dynamic legged robots, in Proceedings of Robotics: Science and Systems (RSS), (Cambridge, Massachusetts, 2017).

14. M. Camurri, M. Fallon, S. Bazeille, A. Radulescu, V. Barasuol, D. G. Caldwell and C. Semini, IEEE Robotics and Automation Society Letters 2, 1023(April 2017).

15. J. Ma, M. Bajracharya, S. Susca, L. Matthies and M. Malchano, International Journal of Robotics Research 35, 631 (2016).

16. A. Chilian, H. Hirschmüller and M. Görner, Multisensor data fusion for robust pose estimation of a six-legged walking robot, in IEEE/RSJ International Conference on Intelligent Robots and Systems (IROS), (San Francisco, CA, 2011).

17. S. Chitta, P. Vernaza, R. Geykhman and D. D. Lee, Proprioceptive localilzatilon for a quadrupedal robot on known terrain, in IEEE International Conference on Robotics and Automation (ICRA), (Roma, Italy, 2007).

18. M. Bloesch, C. Gehring, P. Fankhauser, M. Hutter, M. A. Hoepflinger and R. Siegwart, State estimation for legged robots on unstable and slippery terrain, in IEEE/RSJ International Conference on Intelligent Robots and Systems (IROS), (Tokyo, Japan, 2013).

19. T. Flayols, A. Del Prete, P. Wensing, A. Mifsud, M. Benallegue and O. Stasse, Experimental evaluation of simple estimators for humanoid robots, in IEEE/RAS International Conferences on Humanoid Robots (Humanoids), (Birmingham, UK, 2017).

20. G. Fink and C. Semini, Proprioceptive sensor fusion for quadruped robot state estimation, in (Submitted), (Las Vegas, Nevada, 2020).

21. A. Geiger, P. Lenz and R. Urtasun, Are we ready for autonomous driving? the KITTI vision benchmark suite, in IEEE/CVF Conference on Computer Vision and Pattern Recognition (CVPR), (Providence, RI, 2012).

22. D. Schubert, T. Goll, N. Demmel, V. Usenko, J. Stückler and D. Cremers, The TUM VI benchmark for evaluating visual-inertial odometry, in IEEE/RSJ International Conference on Intelligent Robots and Systems (IROS), (Madrid, Spain, 2018).

23. M. Burri, J. Nikolic, P. Gohl, T. Schneider, J. Rehder, S. Omari, M. W. Achtelik and R. Siegwart, International Journal of Robotics Research 35, 1157 (2016).

24. O. Lamarre, O. Limoyo, F. Marić and J. Kelly, International Journal of Robotics Research $\mathbf{0}$, $1(2020)$.

25. J. Delmerico and D. Scaramuzza, A benchmark comparison of monocular visual-inertial odometry algorithms for flying robots, in IEEE International Conference on Robotics and Automation (ICRA), (Brisbane, Australia, 2018).

26. A. Geiger, P. Lenz, C. Stiller and R. Urtasun, International Journal of Robotics Research 32, 1231 (2013).

27. G. Fink and C. Semini, Proprioceptive sensor dataset for quadrupeds, in Workshop on VisualInertial Navigation: Challenges and Applications, (Macao, China, 2019).

28. C. Semini, HyQ - design and development of a hydraulically actuated quadruped robot, PhD thesis, University of Genoa and Italian Institute of Technology (IIT), (Genoa, Italy, 2010).

29. H. Khan, M. D'Imperio, F. Cannella, D. G. Caldwell, A. Cuschieri and C. Semini, Sensors 17, 1 (2017)

30. R. Mahony, T. Hamel and J. Pflimlin, IEEE Trans. Autom. Control 53, 1203(June 2008).

31. G. Fink, Proprioceptive Sensor Dataset for Quadruped Robots (IEEE Dataport, 2019).

32. S. Fahmi, M. Focchi, A. Radulescu, G. Fink, V. Barasuol and C. Semini, IEEE Transactions on Robotics 36, 443(April 2020). 